\title{
The impact of SUZAKU on the knowledge of CVs and expectation to ASTRO-H
}

\author{
Takayuki Hayashi* \\ Nagoya University, Furo-Cho, Chikusa-ku, Nagoya 464-8602, Japan \\ NASA, Goddard Space Flight Center, Code 662, Greenbelt, MD20771, USA \\ E-mail: thayashi@u.phys.nagoya-u.ac.jp
}

\begin{abstract}
Suzaku was lunched on July 2005 and completed its scientific observations in June 2015. It has advantage especially in the Fe $\mathrm{K}$ shell band with its low background, large effective area and medium energy resolution, and hard X-ray above $10 \mathrm{keV}$ with high sensitivity. In CH Cyg, drastically varying fluorescent $\mathrm{Fe} \mathrm{K}_{\alpha}$ line was detected. The temporal extraordinary large equivalent width of $\sim 1.4 \mathrm{keV}$ is considered to be caused by scattered X-rays and a occultation of the intrinsic source. Plasmas in quiescence and outburst of SS Cyg are surrounded by reflectors with solid angles of $\Omega /(2 \pi)=1.7 \pm 0.3$ and $0.9_{-0.4}^{+0.5}$, respectively. The quiescence case is consistent with the traditional boundary layer, however, the outburst case implies that the plasma locates on the extensive flat reflector. Non-thermal X-ray was detected from nova for the first time in V2491 Cyg 9 days after the nova event, whose spectrum can be reproduced by power-law of photon index of $\Gamma=0.1 \pm 0.2$. In V1223 Sgr, energy centroid synchronizing with the WD spin was detected in the fluorescent $\mathrm{Fe} \mathrm{K}_{\alpha}$ line. It is suggested to be emitted by the pre-shock accreting matter. Recently, thermal and reflection X-ray spectral models of the intermediate polar were updated including variety of the specific accretion rate, dipolar geometry, spherical shape of the reflector and physical quantities distributions in the accretion flow etc.. The WD masses were estimated at $0.91_{-0.03}^{+0.08} M_{\odot}$ and $0.65_{-0.12}^{+0.11} M_{\odot}$ for V1223 Sgr and EX Hya with application of the new models. X-ray observatory ASTRO-H will be launched in February 2016. It will be accomplish energy resolution of $\sim 7 \mathrm{eV}$ at $6 \mathrm{keV}$ with the SXS system, high sensitivity and large effective area measurement above $10 \mathrm{keV}$ by the HXI system and large field of view observation $\left(38 \times 38 \mathrm{arcmin}^{2}\right)$ with the SXI system. They will enable us to directly diagnose plasma density with the triplet of the He-like ions, the detailed gaseous dynamics analysis, geometrical inspection and more frequent serendipitous source detection.
\end{abstract}

The Golden Age of Cataclysmic Variables and Related Objects - III, Golden2015

7-12 September 2015

Palermo, Italy

${ }^{*}$ Speaker. 


\section{Introduction}

Cataclysmic variables (CVs) are binary systems composed of a white dwarf (WD) and a mass donor of late-type main-sequence star filling the Roche-lobe. They emit X-ray via thermal plasma with aid of the WD's gravitational potential. The nature of the systems is drastically changed by the magnetic field strength of the WD. CVs whose WD magnetic field too weak $(B<0.1 \mathrm{MG})$ to control the accreting matter are called non-magnetic CV. In these system, the accretion disk extends close the WD surface. The accreting matter precipitously decelerates in a boundary layer between the innermost of the accretion disk and the WD surface. Then the gravitational potential stored in the Kepler motion is released, and the accreting matter is heated up to order of $10^{8} \mathrm{~K}$ and highly ionized. By contrast, when $B>0.1 \mathrm{MG}$ the systems are called magnetic CVs where the WD magnetic field strong enough to control the accreting matter. The accreting matter is channeled and falls onto the WD along the magnetic field within the Alfvèn radius [28, 3]. The channeled accreting matter falls at almost free fall velocity and heated up and highly ionized via strong shock near the WD surface $[12,1]$. The maximum temperature of the plasma in the magnetic CVs is approximately twice higher than that of the non-magnetic $\mathrm{CVs}$ because the half of the gravitational potential is exhausted in the Keplerian accretion disk in the non-magnetic CVs.

WD binaries hold a red giant instead of the late-type star are called symbiotic star (note that some symbiotic stars hold not a WD but a neutron star [19, 20]). Many symbiotic stars are soft $\mathrm{X}$-ray emitters where the hot WD or optically thin plasma of $\sim 10^{7} \mathrm{~K}$ is thought to be the origin. However, a handful of the WD symbiotic stars emits hard X-ray $(\sim 10 \mathrm{keV})$ whose origin is debatable.

X-ray observations may reveal various natures of the WD binaries, for example, physical quantities of the highly ionized plasma and gases irradiated by the X-ray from the plasma. The plasma temperature gives us depth of the gravitational potential which is used for the WD mass estimation. Scattered and reemitted X-ray from the irradiated gases allow us to inspect geometries of the systems [6]. X-ray can be also originated by non-thermal radiation from high energy electrons.

Suzaku X-ray observatory [21] has some instruments favorable for the WD binary observations. Suzaku equips X-ray CCDs coupled with X-ray telescopes, which have medium energy resolution, large effective area and low background noise. Many of the WD binaries have prominent $\mathrm{Fe} \mathrm{K}$ emission lines of low to high ionization plasma which give us plasma temperature and dynamics of the accreting matter etc.. A high sensitivity hard X-ray $(>10 \mathrm{keV})$ detector is also mounted on Suzaku. It is useful for estimation of the maximum plasma temperature and scattered $\mathrm{X}$-ray.

In February 2016, ASTRO-H observatory [37] will be launched. It has some advantages comparing to X-ray observatory so far. Energy resolution of a micro-calorimeter onboard ASTRO-H is $\sim 50$ times better than that of X-ray CCDs. Imaging capability for the hard X-ray is achieved by extent of effective energy band of X-ray telescope, which is drastically improve the signal-noise ratio. Moreover, the total effective area of ASTRO-H at the Fe K-shell band is largest so far.

In $\S 2$, Suzaku observatory is introduced and in $\S 3$ some Suzaku results are picked up. ASTRO$\mathrm{H}$ observatory and its expectation are introduced in $\S 4$. In $\S 5$, I summarize above. 


\section{Suzaku observatory}

Suzaku observatory is the fifth Japanese X-ray astronomical satellite and collaboration mission between Japan and the United States. It was launched at the Uchinoura Space Center of the Japan Aerospace Exploration Agency on July 10, 2005. The weight and the length of its spacecraft are 1.7 tons and $6.5 \mathrm{~m}$, respectively. Suzaku orbits around the Earth with the period of 96 minutes at $570 \mathrm{~km}$ altitude with an inclination angle of 32 degree and its pointing accuracy is about 0.2 arcmin. Suzaku has four X-ray CCDs called the X-ray Imaging Spectrometer (XIS) which is installed at foci of dedicated X-ray telescope (XRT) for each of them. The Hard X-ray Detector (HXD) onboard Suzaku actualize high sensitivity observation in hard X-ray above $10 \mathrm{keV}$. Suzaku completed its scientific operation in June 2015, however, high quality archival data had been accumulated.

Four modules of the XIS are designated as XIS0-3 [17]. Of them, XIS1 adopts a backilluminated (BI) CCD while the others employ front-illuminated (FI) CCDs. The FI and BI CCDs cover energy ranges of $0.4-10 \mathrm{keV}$ and $0.2-10 \mathrm{keV}$, respectively and their energy resolutions are $\sim 130-250 \mathrm{eV}$ and $\sim 150-300 \mathrm{eV}$, respectively. Energy scale accuracy is typically $\leq 10 \mathrm{eV}$ at the $\mathrm{Mn}-\mathrm{K} \alpha$ line (5.895 keV). After the middle of 2006, the spaced-row charge injection technique was routinely operated which drastically improved the energy resolution [25, 44]. XIS2 and part of XIS0 have been unusable from November 2006 and June 2009, respectively, because of anomalies.

Each XIS module is located in the focal plane of a dedicated X-ray telescope (XRT) module [33]. The XRT adopts Wolter-I type grazing-incident reflective optics consisting of tightly nested, thin-foil conical mirror shells. The angular resolution ranges from 1.8 to $2.3 \mathrm{arcmin}$ in half-power diameter. The effective area is $440 \mathrm{~cm}^{2}$ at $1.5 \mathrm{keV}$ and $250 \mathrm{~cm}^{2}$ at $8 \mathrm{keV}$ per module.

HXD cover energy above $10 \mathrm{keV}$, which is a non-imaging and collimated detector [36, 16]. It is composed of two types of detectors. One is the PIN detector which adopts 2 mm-thick silicon PIN diodes, and is sensitive in up to $70 \mathrm{keV}$. The other is the GSO/BGO phoswitch counter which is sensitive in the 40-600 keV band. The energy resolution is $3.0 \mathrm{keV}$ (FWHM) to the PIN detector, and $7.6 \sqrt{E} \%$ (FWHM) to the GSO detector, where $E$ is energy in MeV. Since in general no significant flux can be detected with the GSO in the WD binaries observation.

\section{Suzaku results}

\subsection{Variation of fluorescent $\mathrm{Fe} \mathrm{K}_{\alpha}$ line in $\mathrm{CH}$ Cygni}

CH Cyg is one of a few WD symbiotic stars known to emit hard X-ray [5]. CH Cyg has produced radio [41] and optical [34] jets, direction of which indicates that its inclination angle is about 90 degree, that is, edge-on. This target was observed by several X-ray observatories (e.g. ASCA in 1994 October, CHANDRA in 2001 March). Suzaku observations were performed in January and May 2006 and summarized in [22].

Figure 1 shows spectra around $\mathrm{Fe} \mathrm{K}_{\alpha}$ lines of $\mathrm{CH}$ Cyg observed by the several X-ray observatories from 1994 to 2006 [22]. These spectra can be fitted with an absorbed bremsstrahlung and three Gaussians. In the Suzaku spectra, the fluorescent line is prominent and its equivalent widths (EWs) are unusually large and $0.7-1.3 \mathrm{keV}$. The fluorescent line was detected with ASCA in 1994, however, its EW is ordinary and $\sim 140 \mathrm{eV}$. Moreover, CHANDRA was not detected this line in 2001. 
These results show that geometry of cool gases originating the fluorescent line is varied. The unusual large EW of the fluorescent line indicates that the intrinsic X-ray source, which irradiated the cool gases, should be hidden with a optically thick material [14] when Suzaku observed. The authors of [22] support a thought that the hot plasma emitting intrinsic hard X-ray is in the boundary layer [5] because of similarity of the temperature and the luminosity $\left(\sim 10^{33} \mathrm{erg} \mathrm{s}^{-1}\right)$ between ASCA observation and a old nova, V603 Aql [23]. They discarded the secondary star of M giant for the blocking material because of the binary ephemeris and considered it likely that the blockage was caused by the disk-edge of accretion disk which is varies from epoch to epoch.

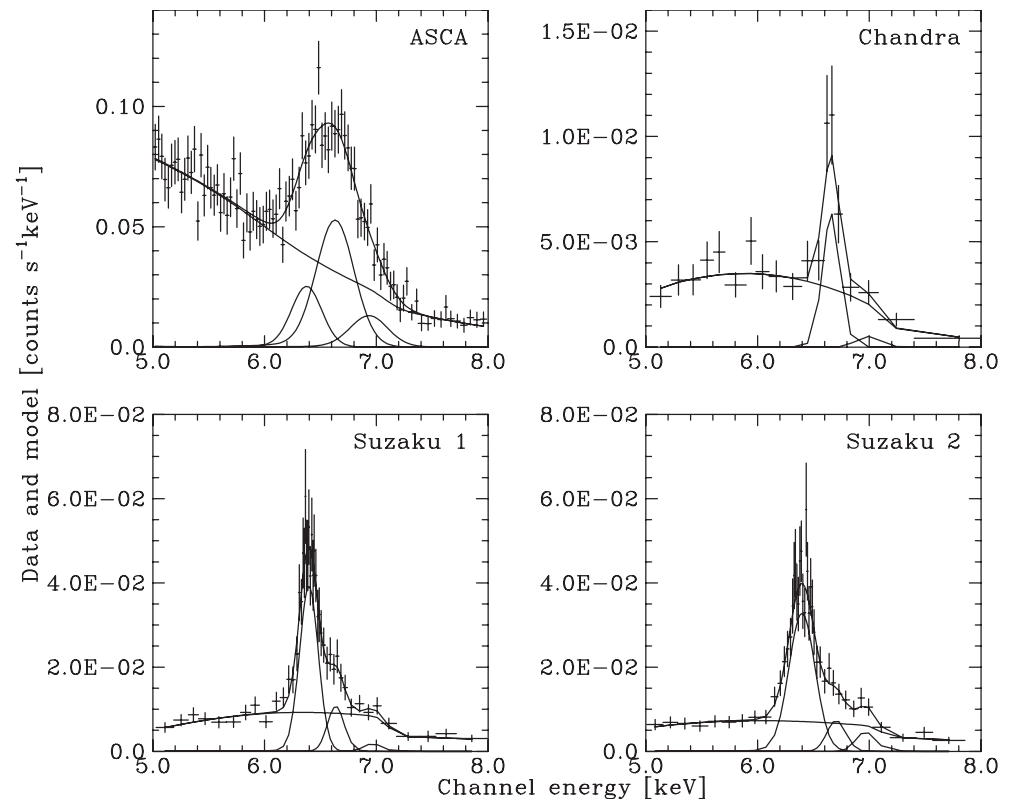

Figure 1: $\mathrm{Fe} \mathrm{K}_{\alpha}$ lines of CH Cyg in 4 observations. ASCA SIS, Chandra ACIS-S (zeroth-order) and Suzaku combined FIs data are shown along with absorbed bremsstrahlung plus Gaussian fits (reprint from [22]).

\subsection{Plasma geometry of SS Cygni in quiescence and outburst}

SS Cyg is one of the most famous dwarf novae, which shows an optical outburst roughly every 50 days. In outburst, the boundary layer is believed to be optically thick and the origin of a hard X-ray optically thin thermal emission is not clear. The optically thin-to-thick transition of boundary layer has been clearly detected with coordinated observations of optical, EUVE, and RXTE [45]. Suzaku observations in both of quiescence and outburst were performed in November 2005, whose results are reported in [15].

In general, dwarf novae has reflection X-ray which gives us hints of geometry and especially a solid angle of reflectors viewing from plasma emitting the intrinsic X-ray [6]. Left panel of figure 2 is theoretical spectra of SS Cyg which shows that the reflection component is comparable to the intrinsic emission at fluorescent $\mathrm{Fe} \mathrm{K}_{\alpha}$ line of $6.4 \mathrm{keV}$ and Compton hump around $10-40 \mathrm{keV}$. The authors of [15] iterated spectral fitting to match the solid angles estimated by the florescent Fe line and the Compton hump [18] and then obtained models reproducing the SS Cyg spectra in the both states as shown in right panel of figure 2 for the quiescence case. The solid angles are estimated at $\Omega /(2 \pi)=1.7 \pm 0.3$ and $0.9_{-0.4}^{+0.5}$ in the quiescence and the outburst, respectively. 
In case of the quiescence, the estimated solid angle is consistent with 1.5, which is thought to indicates that the plasma is surrounded by both of the WD surface and accretion disk and is consistent with the traditional boundary layer. By contrast, the solid angle of the outburst is consistent with 1 , which means that the plasma is on a extensive plane. The plasma is considered to locate on the accretion disk because the fluorescent Fe line is broad with $0.12 \mathrm{keV}$ of $\sigma$ of the fitted Gaussian in the outburst. The authors suggested disk corona for the origin of the plasma in outburst.
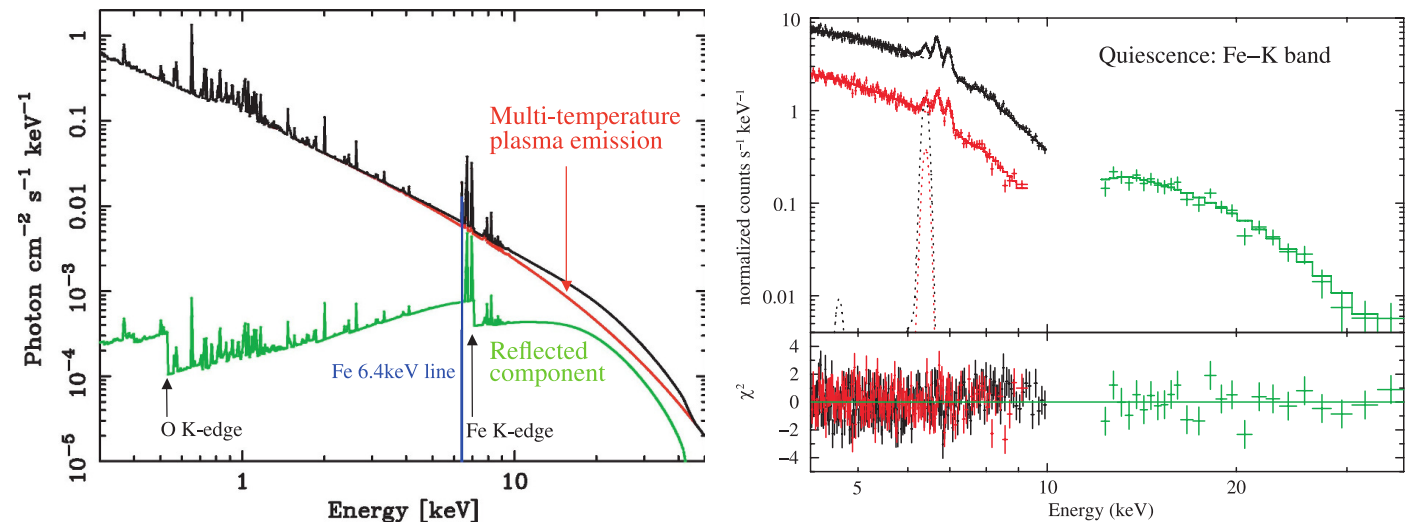

Figure 2: Left : Schematic view of the theoretical spectra of SS Cyg in the $0.2-40 \mathrm{keV}$. Right : Result of fits of model including thermal plasma and reflection to the $4.2-40 \mathrm{keV}$ band in quiescence. Black, red and green are used for the data and the model of the FI-CCD, BI-CCD, and PIN, respectively. (Reprint from [15])

\subsection{Non-thermal X-ray detection from the classical nova V2491 Cygni}

V2491 Cyg [26, 32] was discovered on 2008 April 10.728 UT. The evolution of the nova was extremely fast [42], declining at a rate of t2 4.6 d [43], where t 2 is the time to fade by 2 mag from the optical maximum. In general, classical novae monotonically decline in optical brightness, however V2491 Cyg rebrightened and followed by a sudden fading around day 15. It is suggested that the rebrightening is caused by a sudden release of magnetic energy [7]. Moreover, X-ray with possible spectral changes were detected [13], which also suggests that V2491 Cyg might host a magnetic WD. Suzaku observations were performed from 9.1 (2008 April 19) and 28.9 days (May 9) after the nova event with $20 \mathrm{ks}$ each, whose results were reported by [40, 39].

Figure 3 is Suzaku spectra on 9 day with some fitted models. The source became much dimmer and softer on 29 day. The spectrum on 9 day was very hard and significant signal was detected up to $70 \mathrm{keV}$. On the other hand, a emission line of $6.6 \pm 0.1 \mathrm{keV}$ with $\mathrm{EW}$ of $\sim 240 \mathrm{eV}$ was detected, which is thought to be due to He-like $\mathrm{Fe} \mathrm{K}_{\alpha}$ line. Absence of the H-like Fe line indicates temperature of the plasma is $\sim 10 \mathrm{keV}$, which is incapable of producing the superhard continuum. Authors of [40] performed model fitting with two models; one is optically thin thermal plasma plus a power-law and the other is optically thin thermal plasma plus a bremsstrahlung. They obtained a statistically acceptable fit using the model including power-law with temperature of the thermal component of $2.9_{-2.6}^{+4.3} \mathrm{keV}$ and photon index of the power-law $\Gamma=0.1 \pm 0.2$ (solid lines in figure 3). By contrast, the model using bremsstrahlung even with $100 \mathrm{keV}$ can not reproduce the superhard continuum (blue thin line in figure 3). 
The power-law emission from V2491 Cyg suggests presence of an accelerated population of electrons and is the first to claim a nonthermal signature from classical nova events in the X-ray band although radio interferometer found a evidence of synchrotron radiation [31]. The very hard power-law component with $\Gamma=0.1 \pm 0.2$ needs electron energy distribution of power-law with -0.8 to -1.8 and -0.4 to 1.1 for inverse Compton or synchrotron radiation, and bremsstrahlung, respectively, which are too hard to standard diffusive shock acceleration $(\sim 2.0)$ [2].

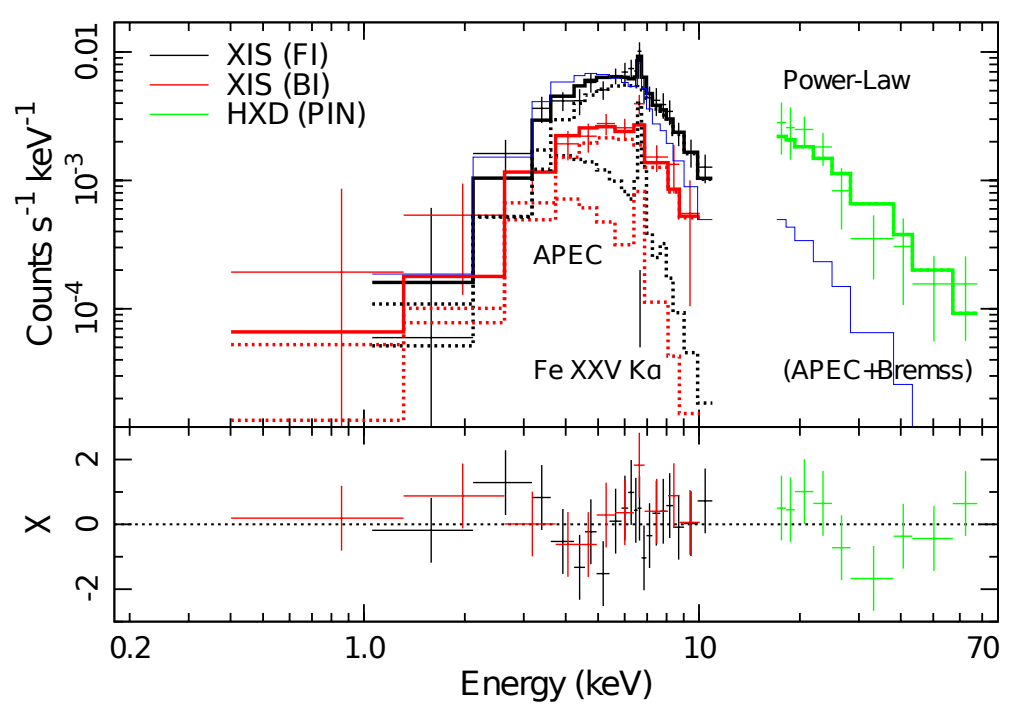

Figure 3: XIS and PIN spectra on day 9 and fitted models. The best-fit model (APEC + power-law) is shown with thick lines (solid for total and dashed for each component) in black, red and green for FI, BI, and PIN, respectively. The alternative unsuccessful model (APEC $+100 \mathrm{keV}$ bremsstrahlung) is shown with thin blue lines. The lower panel shows the residual from the best fit. (Reprint from [40])

\subsection{Line centroid modulation in V1223 Sagittarii}

In general, X-ray intensity of intermediate polars (IPs) modulates with their spin period, where IP is a subclass of $\mathrm{mCV}$ whose WD's magnetic field is moderate $(0.1<B<10 \mathrm{MG})$. The X-ray modulation is thought to be caused mainly by photo-electric absorption with pre-shock accreting matter, which implies that X-ray is less intense at pole-on [30]. V1223 Sgr is a typical IP and its $\mathrm{X}$-ray also modulate with the spin period of 745.6s [27]. Suzaku observation of V1223 Sgr was performed in April 2007 and the results were reported by [10].

Left panel of figure 4 shows X-ray intensity and energy centroid of the fluorescent $\mathrm{Fe} \mathrm{K}_{\alpha}$ line in two spin periods. The energy centroid of the fluorescent $\mathrm{Fe}_{\alpha}$ line is clearly modulated with the spin period as well as the $\mathrm{X}$-ray intensity, and the energy centroid is lowest when X-ray intensity is minimum. The discrepancy of the energy centroid from $6.4 \mathrm{keV}$ of the nominal energy is $\sim 20 \mathrm{eV}$ at the X-ray intensity-minimum phase and the EW of that line is $\sim 100 \mathrm{eV}$.

The fluorescent $\mathrm{Fe} \mathrm{K}_{\alpha}$ line can be divided into two narrow components as shown in right panel of figure 4 at the intensity-minimum phase. The energy centroid of one of them is fixed at $6.4 \mathrm{keV}$ and its EW estimated at $\sim 80 \mathrm{eV}$. On the other hand, the energy centroid of the other was freed and converged on $6.30_{-0.05}^{+0.07} \mathrm{keV}$ and its EW was estimated at $\sim 30 \mathrm{eV}$. The EW of the $6.4 \mathrm{keV}$ and the $6.3 \mathrm{keV}$ components are consistent with the predictions of reflection from the WD surface 
[6] and hydrogen column density [14] of $12.3_{-2.5}^{+1.7} \mathrm{~cm}^{-2}$ obtained by global X-ray spectral fitting, respectively. Moreover, velocity calculated with the energy discrepancy from $6.4 \mathrm{keV}$ is $4.7_{-3.3}^{+2.3} \times$ $10^{3} \mathrm{~km} \mathrm{~s}^{-1}$, which is roughly consistent with free fall velocity at the WD of $(5.6 \pm 0.4) \times 10^{3} \mathrm{~km}$ $\mathrm{s}^{-1}$ assuming the WD mass is $0.82_{-0.06}^{+0.05} M_{\odot}$ estimated by X-ray spectrum. These results suggest that the $6.3 \mathrm{keV}$ component is emitted by the pre-shock accreting matter, which is consistent with the model of the X-ray modulation caused by the pre-shock accreting matter.
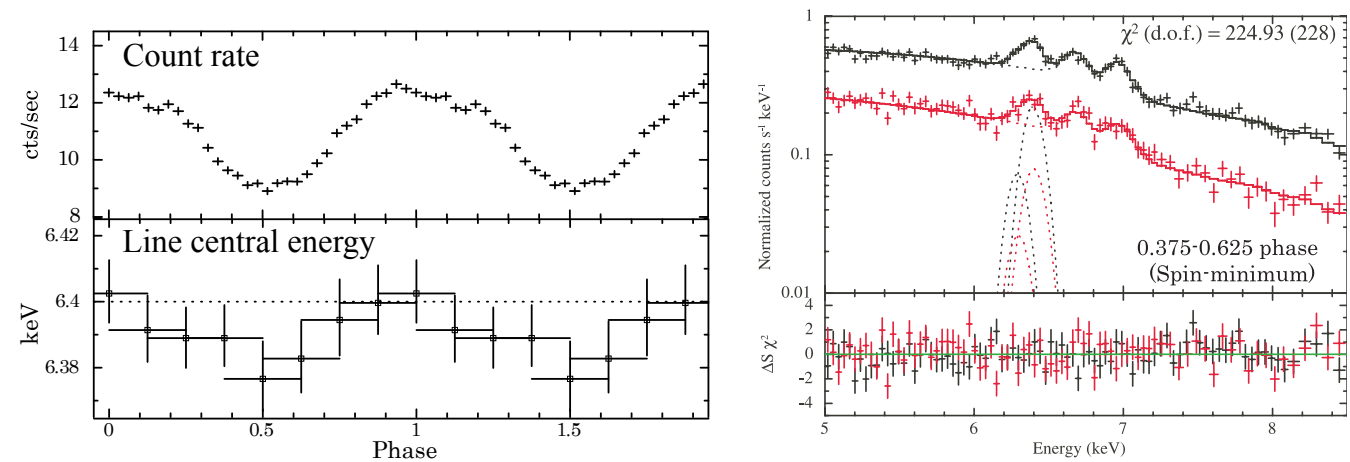

Figure 4: Left panel : Folded light curve at the period of $745.7 \mathrm{~s}$ (top) and energy centroid of fluorescent $\mathrm{Fe} \mathrm{K}_{\alpha}$ in corresponding spin phase (bottom). Right : The $5-8.5 \mathrm{keV}$ spectra at the intensity-minimum phase $0.357-0.625$ fixed fitted with two Gaussians. (Reprint from [10])

\subsection{Update of X-ray spectral model for IP}

A lot of thermal X-ray spectral models of IPs were constructed, which have been used for the WD mass estimation. The post-shock accretion flow in magnetic CV is somewhat simple comparing with the accretion disk and can be approximated by quasi-one-dimensional flow. Moreover, cyclotron cooling of IPs is considered to be ignored [46]. Therefore, many hydrodynamical models were proposed for the IPs and thermal X-ray spectral models were constructed based on the hydrodynamical models (e.g. $[1,4,35])$. Recently, accretion rate per unit area called specific accretion rate was parameterized with including dipolar geometry for the accretion flow [8,9]. Note that the variety of the specific accretion rate was included in [4], however, after that, the specific accretion rate had been inclined to be fixed at a typical value of $1 \mathrm{~g} \mathrm{~cm}^{-2} \mathrm{~s}^{-1}$ (e.g. [35]).

By contrast, reflected X-ray by the WD was somewhat poorly modeled. The reflected X-ray plays a important roll in the IPs as well as dwarf novae (\$3.2). We performed a Monte-Carlo simulation in order to model the reflected X-ray spectrum with a cool and spherical WD and accretion flows as X-ray sources in which physical quantities distributions are involved. A part of photons emitted from the source arrived at the WD and they undergo a sequence of interactions of scattering, photoelectric absorption and reemission of $\mathrm{K}_{\alpha}$ and $\mathrm{K}_{\beta}$ from neutral $\mathrm{Fe}$ and nickel (see [11] for more detail). A part of the arrived photon escapes from the WD after the interaction sequence.

Left panel of figure 5 is a example of X-ray spectrum calculated with the updated thermal and reflected X-ray spectral models. With a more massive WD, the thermal component becomes harder. On the oner hand, the more massive WD makes more intense Compton hump and larger EW of the reemitted lines because higher energy photon has more chance to escape and absorption by K-shell of Fe or nickel. Note that the reflection spectrum depends on a viewing angle between a line along 
the accretion flow and line of sight mainly because optical path is longer to photon escaped with the larger viewing angle. We performed model fitting to Suzaku data of V1223 Sgr and EX Hya with the new X-ray spectral models as shown in right panel of figure 5. As a results, we obtained WD masses of $0.91_{-0.03}^{+0.08} M_{\odot}$ and $0.65_{-0.12}^{+0.11} M_{\odot}$ to V1223 Sgr and EX Hya, respectively.
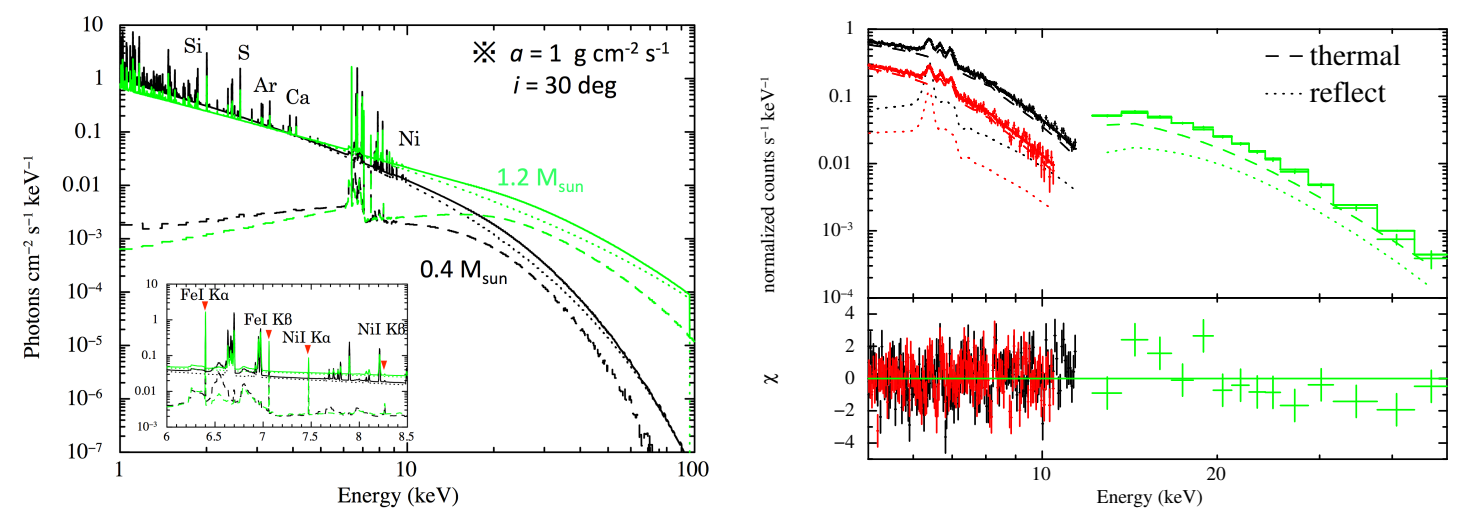

Figure 5: Left panel : X-ray spectral model combined the updated thermal model and the new reflection model. Solid, dotted and dashed lines are total, thermal and reflection components, respectively. The WD masses of $0.4 M_{\odot}$ and $1.2 M_{\odot}$ are assumed in black and green, respectively. The specific accretion rate and viewing angle are assumed at $1 \mathrm{~g} \mathrm{~cm}^{-2} \mathrm{~s}^{-1}$ and $30 \mathrm{deg}$, respectively, in this figure. Right panel : V1223 Sgr X-ray spectra observed by Suzaku together with the fitted model with the combined X-ray spectral model.

\section{ASTRO-H expectation}

\subsection{ASTRO-H observatory}

ASTRO-H satellite will be launched in February 2016 [38, 48]. Three observational systems are equipped in ASTRO-H which are available in a energy band important for the WD binaries, that is, below $100 \mathrm{keV}$. One of them is a couple of Soft X-ray Telescope (SXT) and Soft X-ray Spectrometer (SXS), which has significant effective area in $0.3-12 \mathrm{keV}$. The SXS is micro-calorimeter and its energy resolution is $\sim 7 \mathrm{eV}$ at $6 \mathrm{keV}$ which is highest around the Fe $\mathrm{K}$ shell band. The SXS system has effective area of $\sim 225 \mathrm{~cm}^{2}$ at $8 \mathrm{keV}$ with aid of SXT. Two hard X-ray imagers (HXIs) coupled with Hard X-ray Telescope (HXT) have imaging capability from $5 \mathrm{keV}$ to $80 \mathrm{keV}$. Each of the HXT systems have a effective area of $300 \mathrm{~cm}^{2}$ at $30 \mathrm{keV}$. The other system is Soft X-ray Imager (SXI) coupled with SXT which has large field of view (FOV) of $38 \times 38 \mathrm{arcmin}^{2}$.

The high energy resolution of the SXS systems will provide various important informations. For example, triplet of the Fe He-like ion will be resolved which allows us directly to diagnose plasma density [24]. Line energy measurement with SXS gives us detailed gaseous dynamics. The fluorescent lines give us geometrical informations with their EWs and Compton shoulder shape.

High sensitivity and large effective area of the HXI systems will make it possible clearly to divide the thermal and the reflection components. The accurate measurement of the thermal and reflection components in the hard X-ray are important to decide the maximum plasma temperature and unveil the geometry. 
Large FOV of the SXI system will enhance possibility of serendipitous sources detection. Image of SXI will be helpful for estimation of contamination into SXS from nearby sources.

\subsection{Expectation of V1223 Sgr observation with ASTRO-H}

V1223 Sgr will be observed by ASTRO-H in the performance verification (PV) phase with $100 \mathrm{ks}$ (see [49] for other targets). V1223 Sgr is a typical and one of the brightest IP (flux in 2$10 \mathrm{keV} \sim 9.52 \times 10^{11} \mathrm{erg} \mathrm{cm}^{-2} \mathrm{~s}^{-1}$ ), which is intensely observed with previous X-ray observatories (e.g. [10]). Expectation of the V1223 Sgr observation with ASTRO-H is summarized in [24] in detail.

Left panel of figure 6 is expected spin-phase-resolved spectra taken with SXI and HXI systems each of whose exposure is $20 \mathrm{ks}$. Intensity and shape of the Compton hump of the reflection component varies with the viewing angle and the viewing angle will be able to be measured with accuracy of $\cos \theta=0.2$ at least using only the Compton hump.

In general, density of the plasma in the IPs is thought to be included in a range in which the He-like triplet of Fe has sensitivity to the density $\left(\sim 10^{17-18} \mathrm{~cm}^{-3}\right)$. As shown in right panel of figure 6 , especially intensities of the forbidden and intercombination lines vary with the plasma density [29] characterized by $f$. $f$ is a ratio of accretion flow cross-section to the WD surface area. When the total accretion rate is fixed at $8.4 \times 10^{16} \mathrm{~g} \mathrm{~s}^{-1}, \mathrm{f}=0.0002$ and 0.005 correspond to 54.2 and $2.18 \times 10^{16} \mathrm{~cm}^{-3}$, respectively, at the post-shock region. The direct density diagnostics will polish up the hydrodynamical model of the accretion flow and enhance the accuracy of the WD mass estimation.
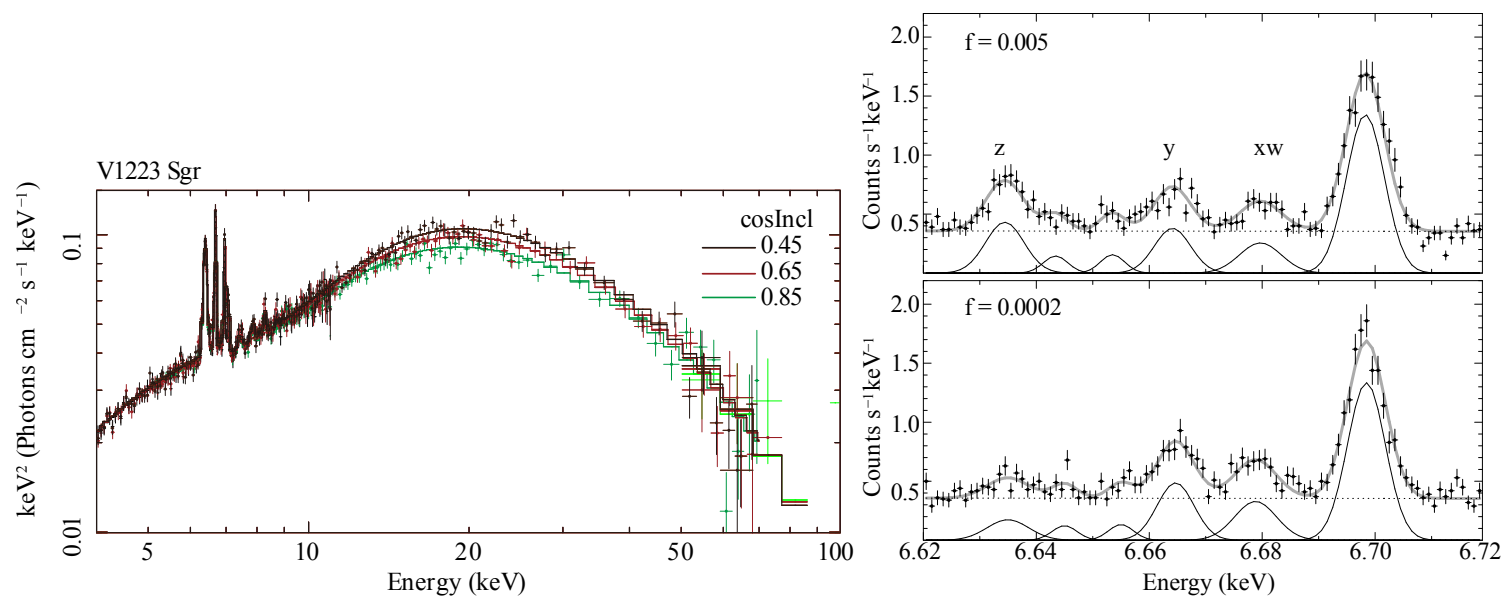

Figure 6: Left : Expected spin-phase-resolved SXI and HXI $v F v$ spectra of V1223 Sgr assuming the viewing angle $\cos \theta=0.45,0.65$, and 0.85 with $20 \mathrm{~s}$ each (total is $100 \mathrm{ks}$ ). Right : Expected He-like Fe $\mathrm{K}_{\alpha}$ emission line of V1223 Sgr observed with the SXS system assuming $100 \mathrm{ks}$ exposure. Two cases of covering fractions $f=0.005$ and 0.0002 are shown. (reprint from [24])

\section{Summary}

Suzaku observatory was lunched on July 2005 and completed its scientific observations in June 2015. It has advantage to the WD binary observation comparing to other observatories in the Fe K 
shell band with low background noise, large effective area and medium energy resolution, and the hard X-ray region above $10 \mathrm{keV}$ with high sensitivity.

In $\mathrm{CH}$ Cyg, the fluorescent $\mathrm{Fe} \mathrm{K}_{\alpha}$ line varying in its $\mathrm{EW}$ were clearly detected with some X-ray observatories including Suzaku. Its EW drastically varied from $\sim 0$ to $1.4 \mathrm{keV}$. The extraordinary large EW is considered to be caused by scattered X-ray with a occulted direct source.

SS Cyg in quiescence and outburst were observed by Suzaku with which geometries of the both states were inspected using fluorescent $\mathrm{Fe}_{\alpha} \mathrm{K}_{\alpha}$ line and Compton hump. In quiescence, the plasma is surrounded by a optically thick reflector with a solid angle of $\Omega /(2 \pi)=1.7 \pm 0.3$, which is consistent with the traditional boundary layer. By contrast, in outburst, the solid angle of the reflector was estimated at $\Omega /(2 \pi)=0.9_{-0.4}^{+0.5}$, which implies that the plasma locates on a extensive flat reflector. Disk corona was suggested as a origin of the plasma in outburst.

Suzaku detected non-thermal X-ray from nova for the first time. In V2491 Cyg, a very hard Xray spectrum was detected 9 days after the nova event. The hard spectrum can not be reproduced by bremsstrahlung of even $100 \mathrm{keV}$ and is reproduced by power-law of photon index of $\Gamma=0.1 \pm 0.2$. The hard photon index is too hard comparing to standard diffusive shock acceleration.

In V1223 Sgr, the fluorescent $\mathrm{Fe} \mathrm{K}_{\alpha}$ line whose central energy shifts synchronizing with the WD spin was detected with Suzaku. The shift amount becomes the largest $(\sim 20 \mathrm{eV})$ at intensityminimum phase, where the fluorescent line can be divided into components of stable at $6.4 \mathrm{keV}$ and shifting to $6.30_{-0.05}^{+0.07} \mathrm{keV}$. The $\mathrm{EW}$ of the $6.3 \mathrm{keV}$ component agrees with a prediction with hydrogen column density of $12.3_{-2.5}^{+1.7} \mathrm{~cm}^{-2}$. Moreover, the shift amount of $100 \mathrm{eV}$ is roughly consistent with free fall velocity of $(5.6 \pm 0.4) \times 10^{3} \mathrm{~km} \mathrm{~s}^{-1}$ assuming the WD mass is $0.82_{-0.06}^{+0.05} M_{\odot}$. These results suggest that the $6.3 \mathrm{keV}$ component is emitted by the pre-shock accreting matter, which is consistent with the standard picture, that is, pole-on at intensity-minimum phase.

Recently, thermal X-ray spectral model of the IPs was updated with including variety of the specific accretion rate and dipolar geometry etc. into hydrodynamical model of the post-shock accretion flow. Moreover, the X-ray reflection from the WD surface was modeled by Monte-Carlo simulation where a cool and spherical WD as reflector and physical quantities distributions in the accretion flows are considered. These X-ray spectral model were applied to Suzaku data of V1223 Sgr and EX Hya which gives us the WD masses of $0.91_{-0.03}^{+0.08} M_{\odot}$ and $0.65_{-0.12}^{+0.11} M_{\odot}$, respectively.

X-ray observatory ASTRO-H will be launched in February 2016. The high energy resolution of SXS ( $\sim 7 \mathrm{eV}$ at $6 \mathrm{keV}$ ) will be enable us directly to diagnose plasma density with the triplet of the Fe He-like ion. The detailed gaseous dynamics and geometrical analysis will become possible by high accuracy measurement of the energy centroid, the EW and the Compton shoulder shape of various emission lines. High sensitivity and large effective area measurement above $10 \mathrm{keV}$ by the HXI systems will measure the maximum temperature of the plasma and Compton hump with high accuracy dividing the thermal component and the reflection component. They will play important rolls for the WD mass estimation and the geometric inspection. Large FOV of SXI $\left(38 \times 38 \mathrm{arcmin}^{2}\right)$ will give us more chance of detection of the serendipitous sources. V1223 Sgr will be observed by ASTRO-H in the performance verification phase with $100 \mathrm{ks}$.

\section{References}

[1] Aizu, K. 1973, Progress of Theoretical Physics, 49, 1184 
[2] Blandford, R. D., \& Ostriker, J. P. 1980, ApJ, 237, 793

[3] Cropper, M. 1990, SSRv, 54, 195

[4] Cropper, M., Wu, K., Ramsay, G., \& Kocabiyik, A. 1999, MNRAS, 306, 684

[5] Ezuka, H., Ishida, M., \& Makino, F. 1998, ApJ, 499, 388

[6] George, I. M., \& Fabian, A. C. 1991, MNRAS, 249, 352

[7] Hachisu, I., \& Kato, M. 2009, ApJ, 694, L103

[8] Hayashi, T., \& Ishida, M. 2014, MNRAS, 438, 2267

[9] Hayashi, T., \& Ishida, M. 2014, MNRAS, 441, 3718

[10] Hayashi, T., Ishida, M., Terada, Y., Bamba, A., \& Shionome, T. 2011, PASJ, 63, S739

[11] Hayashi, T., Kitaguchi, T \& Ishida, M. 2017, MNRAS

[12] Hōshi, R. 1973, Progress of Theoretical Physics, 49, 776

[13] Ibarra, A., \& Kuulkers, E. 2008, The Astronomer's Telegram, 1473, ATel

[14] Inoue, H. 1985, SSRv, 40, 317

[48] Ishida, M. 2015, proc of The Golden Age of Cataclysmic Variables and Related Objects - III

[15] Ishida, M., Okada, S., Hayashi, T., et al. 2009, PASJ, 61, S77

[16] Kokubun, M., Makishima, K., Takahashi, T., et al. 2007, PASJ, 59, 53

[17] Koyama, K., Tsunemi, H., Dotani, T., et al. 2007, PASJ, 59, 23

[18] Magdziarz, P., \& Zdziarski, A. A. 1995, MNRAS, 273, 837

[19] Masetti, N., Orlandini, M., Palazzi, E., Amati, L., \& Frontera, F. 2006, A\&A, 453, 295

[20] Mattana, F., Götz, D., Falanga, M., et al. 2006, A\&A, 460, L1

[21] Mitsuda, K., Bautz, M., Inoue, H., et al. 2007, PASJ, 59, 1

[49] Mukai, K. 2015, proc of The Golden Age of Cataclysmic Variables and Related Objects - III

[22] Mukai, K., Ishida, M., Kilbourne, C., et al. 2007, PASJ, 59, 177

[23] Mukai, K., \& Orio, M. 2005, ApJ, 622, 602

[24] Mukai, K., Yuasa, T., Harayama, A., et al. 2014, arXiv:1412.1163

[25] Nakajima, H., Yamaguchi, H., Matsumoto, H., et al. 2008, PASJ, 60, S1

[26] Nakano, S., Beize, J., Jin, Z.-W., et al. 2008, IAUC, 8934, 1

[27] Osborne, J. P., Rosen, R., Mason, K. O., \& Beuermann, K. 1985, SSRv, 40, 143

[28] Patterson, J. 1994, PASP, 106, 209

[29] Porquet, D., \& Dubau, J. 2000, A\&AS, 143, 495

[30] Rosen, S. R., Mason, K. O., \& Cordova, F. A. 1988, MNRAS, 231, 549

[31] Rupen, M. P., Mioduszewski, A. J., \& Sokoloski, J. L. 2008, ApJ, 688, 559-567

[32] Samus, N. N. 2008, IAUC, 8934, 2

[33] Serlemitsos, P. J., Soong, Y., Chan, K.-W., et al. 2007, PASJ, 59, 9 
[34] Solf, J. 1987, $A \& A, 180,207$

[35] Suleimanov, V., Revnivtsev, M., \& Ritter, H. 2005, A\&A, 435, 191

[36] Takahashi, T., Abe, K., Endo, M., et al. 2007, PASJ, 59, 35

[37] Takahashi, T., Mitsuda, K., Kelley, R., et al. 2010, SPIE proc, 7732, $77320 Z$

[38] Takahashi, T., Mitsuda, K., Kelley, R., et al. 2014, SPIE proc, 9144, 914425

[39] Takei, D., \& Ness, J.-U. 2010, Astronomische Nachrichten, 331, 183

[40] Takei, D., Tsujimoto, M., Kitamoto, S., et al. 2009, ApJ, 697, L54

[41] Taylor, A. R., Seaquist, E. R., \& Mattei, J. A. 1986, Natur, 319, 38

[42] Tomov, T., Mikolajewski, M., Ragan, E., Swierczynski, E., \& Wychudzki, P. 2008, The Astronomer's Telegram, 1475, ATel

[43] Tomov, T., Mikolajewski, M., Brozek, T., et al. 2008, The Astronomer's Telegram, 1485, ATel

[44] Uchiyama, H., Ozawa, M., Matsumoto, H., et al. 2009, PASJ, 61, S9

[45] Wheatley, P. J., Mauche, C. W., \& Mattei, J. A. 2003, MNRAS, 345, 49

[46] Wu, K., Chanmugam, G., \& Shaviv, G. 1994, ApJ, 426, 664 